\title{
Estimation WorksPace OF 6-DOF HaUfF Platform
}

\author{
Elena S. Gebel
}

Omsk State Technical University, Mira avenue 11, Omsk,644050, Russia

\begin{abstract}
Investigated spatial mechanism with parallel structure is widely used in different mechatronic devices. There are not only advantages of usage the Hauff platform, such as increased rigidity and compactness of design, but also the disadvantages namely a loss of control. To identify the workspace of studied mechanism, the direct kinematic problem is solved and formed the homogeneous transformation matrix. For evaluation of the singular loci in kinematics of 6 DOF Hauff Platform the screw theory is more appropriate than the Jacobian matrix. The singular poses have been estimated through the matrix of Plucker co-ordinates of unit vectors that are coincided with rods of the studied manipulator and their relative moments. The objective of comparison is to illustrate the capability of the described methods to quantitatively evaluation the volume of the workspace without singular loci.
\end{abstract}

Keyword: Parallel mechnism, direct kinematic problem, singular pose, twist.
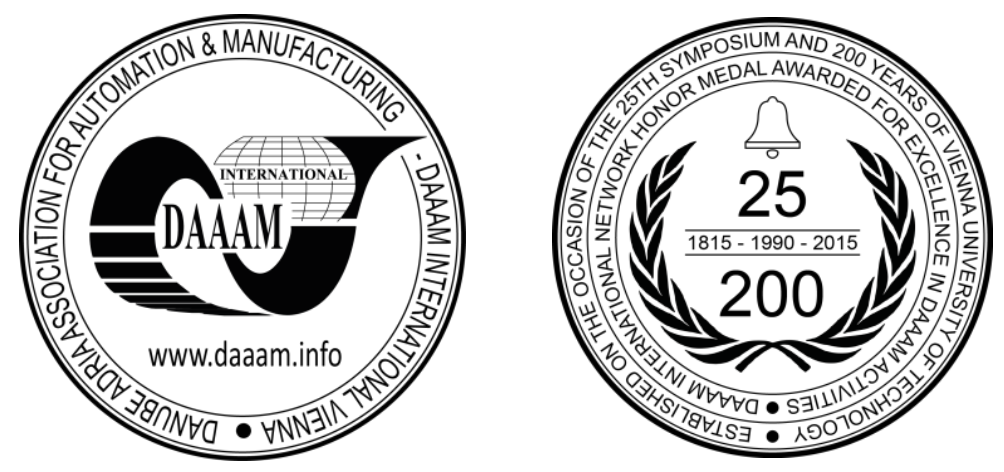

This Publication has to be referred as: Gebel, E[lena] (2016). Estimation Workspace of 6 DOF Hauff platform, Proceedings of the 26th DAAAM International Symposium, pp.0780-0786, B. Katalinic (Ed.), Published by DAAAM International, ISBN 978-3-902734-07-5, ISSN 1726-9679, Vienna, Austria DOI:10.2507/26th.daaam.proceedings.109 


\section{Introduction}

One of the important trends of development of modern robotic systems is the design of mechatronic devices, which provide high precision control of translation and rotation of the output level, as well as the rigidity of the device under dynamic loads. In this regard, it is the most advantageous of usage mechanisms with a parallel structure, such as Hauff platform. Currently, these mechanisms are used as a base component of the table for mounting a cell of segments of the screen of underground tunnels as well as building structures. Also they are applied in the bearing structures of multi-axis machining and assembly equipment and in particular, in the dynamic simulator of truck drivers [12, 13]. In listed devices have been used the different modification of the parallel mechanism which can be distinguished by the various type and quantity of bars as well as the diverse number of degrees of freedom.

Discussed in this paper Hauff platform is depicted in Fig. 1. The position and orientation control of the moving base of the parallel mechanism is realized via change in length of the legs. The main elements of legs are integrally embedded electric drive. A rigid joining of rods with moving and base platform guarantees the absence of "backlash".

The mechanisms with parallel kinematics characterized by the following features:

1. Anisotropy and heterogeneity of dynamic, resilient and high-speed characteristics;

2. Loss of control in some configurations;

3. Interference of separate kinematic chains;

4. Impossibility to derive the control law by the generalized coordinates associated in view of a large number of degrees of freedom.

Thus, one of the major problems concerned with the mechanisms of the parallel structures, in particular with the Hauff platform, is to determine the region of permissible motions (generally screw) of the moving base under the prescribed constrains of the input actions.

Using existed methods for evaluation of singularities of a parallel mechanism, this paper will compare the region of Hauff platforms' workspace for each of them. As a result, it will be done the conclusion which of discussed methods greatly reduces a volume of the mechanism workspace.

\section{Object of study}

The mechanism included in this study is the 6-DOF parallel mechanism [14]. The mechanism, shown schematically in Fig. 1, consists of six identical kinematic chains. Both of the base and moving platform of the mechanism are equilateral triangles which rotate relative to each other at the angle of $60 \square$. Positions of support joints of the upper and lower triangular bases specified by the magnitudes of the circle's radius R1 and R2 correspondently.

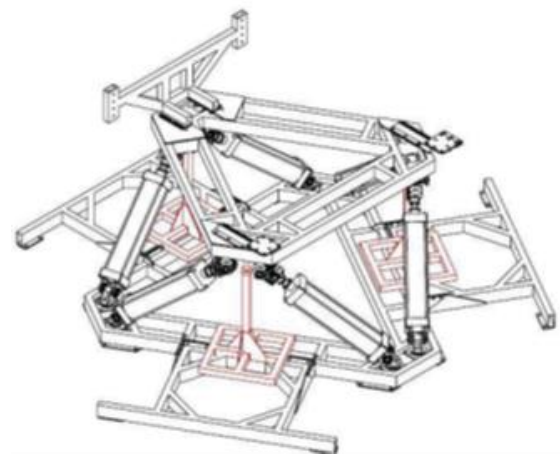

Fig. 1. Platform design

Rods are fixed in pairs by cardan and spherical joints at the vertices of the moving and base triangular platform, respectively. In the study the rods of Hauff platform simulated through the weightless links with a variable length.

\section{Direct kinematics problem}

As a result of direct kinematics study of Hauff manipulator, the position and orientation of the mobile platform and end effector have to determine for the given values of the generalized coordinates namely the lengths of telescopic rods.

In paper [5] it is proved that six values are not enough for an exact evaluation of a body position in space. The solution of the direct kinematics problem of 6-DOF parallel manipulator in this case is not unique. 
Many well-known approaches to solving the kinematic problem of the moving base of a platform can be divided into two groups. Some of them are based on the vector algebra, other ones are used an analytical geometry. In general, the mathematical model of the kinematics of the studied mechanism is a system of nonlinear equations, and for its solution it is required a lot of given magnitude of parameters describing the design features. As shown in paper [10], a system of equations for the 6-DOF parallel manipulator can have up to 40 different possible solutions.

The mathematical model of kinematics of the studied mechanism has been formed through the stationary $O_{1} x_{1} y_{1} z_{1}$ and relative $O_{2} x_{2} y_{2} z_{2}$ cartesian coordinate systems correspondently. The origins of both coordinate systems lie at the intersection of the medians of the equilateral triangles $A_{1} B_{1} C_{1}$ and $A_{2} B_{2} C_{2}$. Axes of these coordinate systems are directed as depicted in Fig. 2.

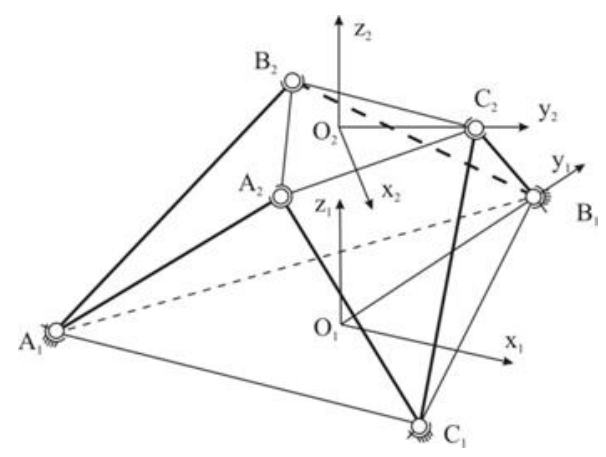

Fig. 2. Kinematic scheme of Hauff Platform

The position and orientation of the moving platform of the studied parallel mechanism are described by vector $X=\left(x_{O 2}, y_{O 2}, z_{O 2}, \alpha, \beta, \gamma\right)$. The first three elements of this vector are the Cartesian coordinates of the center of the moving platform and the last three ones are Euler angles. All of these six parameters uniquely define the orientation of the relative coordinate system $O_{2} x_{2} y_{2} z_{2}$ with respect to the base coordinate system $O_{1} x_{1} y_{1} z_{1}$. As a result of solving the direct kinematics problem will be established a functional relation $X=f(l)$.

In order to calculate six unknown parameters, it is used the equations of holonomic stationary ideal relations between the joints of upper and lower bases which were written through the formulation of Euclidean distance between two points in a space:

$$
\begin{aligned}
& l_{A 1 A 2}^{2}=\left(x_{A 1}-x_{A 2}\right)^{2}+\left(y_{A 1}-y_{A 2}\right)^{2}+\left(z_{A 1}-z_{A 2}\right)^{2} \\
& l_{A 1 B 2}^{2}=\left(x_{A 1}-x_{B 2}\right)^{2}+\left(y_{A 1}-y_{B 2}\right)^{2}+\left(z_{A 1}-z_{B 2}\right)^{2} ; \\
& l_{B 1 B 2}^{2}=\left(x_{B 1}-x_{B 2}\right)^{2}+\left(y_{B 1}-y_{B 2}\right)^{2}+\left(z_{B 1}-z_{B 2}\right)^{2} ; \\
& l_{B 1 C 2}^{2}=\left(x_{B 1}-x_{C 2}\right)^{2}+\left(y_{B 1}-y_{C 2}\right)^{2}+\left(z_{B 1}-z_{C 2}\right)^{2} ; \\
& l_{C 1 C 2}^{2}=\left(x_{C 1}-x_{C 2}\right)^{2}+\left(y_{C 1}-y_{C 2}\right)^{2}+\left(z_{C 1}-z_{C 2}\right)^{2} ; \\
& l_{C 1 A 2}^{2}=\left(x_{C 1}-x_{A 2}\right)^{2}+\left(y_{C 1}-y_{A 2}\right)^{2}+\left(z_{C 1}-z_{A 2}\right)^{2}
\end{aligned}
$$

Coordinates of $A_{1}, B_{1}$ and $C_{1}$ points on the base platform were found through the well-known trigonometric relations for equilateral triangle $\mathrm{A}_{1} \mathrm{~B}_{1} \mathrm{C}_{1}$ :

$$
A_{1}=\left(-\frac{\sqrt{3} R_{1}}{2},-\frac{R_{1}}{2}, 0\right), B_{1}=\left(0, R_{1}, 0\right), C_{1}=\left(\frac{\sqrt{3} R_{1}}{2},-\frac{R_{1}}{2}, 0\right)
$$

Additional equations for calculating the positions of the joins of the moving platform are formed by using the formula of analytic geometry in a space: 


$$
\begin{aligned}
& \left(x_{A 2}-x_{B 2}\right)^{2}+\left(y_{A 2}-y_{B 2}\right)^{2}+\left(z_{A 2}-z_{B 2}\right)^{2}=3 R_{2}^{2} \\
& \left(x_{A 2}-x_{C 2}\right)^{2}+\left(y_{A 2}-y_{C 2}\right)^{2}+\left(z_{A 2}-z_{C 2}\right)^{2}=3 R_{2}^{2} \\
& \left(x_{C 2}-x_{B 2}\right)^{2}+\left(y_{C 2}-y_{B 2}\right)^{2}+\left(z_{C 2}-z_{B 2}\right)^{2}=3 R_{2}^{2}
\end{aligned}
$$

Non-linear system of equations (1) - (9) can be solved through the well-known numerical methods such as Newton's method.

The end effector on the moving platform of Hauff manipulator is fixed at the intersection of the medians of equilateral triangle $A_{2} B_{2} C_{2}$. Thus, using the known relation to calculate the coordinates of the point dividing the segment in a certain ratio, it was written the expression for finding out the center of the coordinate system $\mathrm{O}_{2} x_{2} y_{2} z_{2}$ :

$$
x_{O 2}=\frac{x_{A 2}+x_{B 2}+x_{C 2}}{3} ; y_{O 2}=\frac{y_{A 2}+y_{B 2}+y_{C 2}}{3} ; z_{O 2}=\frac{z_{A 2}+z_{B 2}+z_{C 2}}{3}
$$

o estimate the rotation of axes of coordinate system $\mathrm{O}_{2} x_{2} y_{2} z_{2}$ relative to the axes of stationary coordinate system $O_{1} x_{1} y_{1} z_{1}$, it is formed a homogeneous matrix transformations.

$T=\left[\begin{array}{cccc}n_{x} & s_{x} & a_{x} & x_{O 2} \\ n_{y} & s_{y} & a_{y} & y_{O 2} \\ n_{z} & s_{z} & a_{z} & z_{O 2} \\ 0 & 0 & 0 & 1\end{array}\right]$

Firstly, the matrix equation of the plane passing through three given points $A_{2}, B_{2}$ and $C_{2}$ is written as follows:

$$
\left|\begin{array}{lll}
x-x_{A 2} & x_{B 2}-x_{A 2} & x_{C 2}-x_{A 2} \\
y-y_{A 2} & y_{B 2}-y_{A 2} & y_{C 2}-y_{A 2} \\
z-z_{A 2} & z_{B 2}-z_{A 2} & z_{C 2}-z_{A 2}
\end{array}\right|=0
$$

In general, the equation of plane $A_{2} B_{2} C_{2}$ is obtained by resolving the matrix (11) into the elements of the first column:

$$
\left(x-x_{A 2}\right) \cdot d_{1}-\left(y-y_{A 2}\right) \cdot d_{2}+\left(z-z_{A 2}\right) \cdot d_{3}=0
$$

Variables $d_{i}(i=1, \ldots, 3)$ are calculated from matrix (11) as the determinant of the respective square matrices:

$$
\begin{aligned}
& d_{1}=\left(y_{B 2}-y_{A 2}\right) \cdot\left(z_{C 2}-z_{A 2}\right)-\left(y_{C 2}-y_{A 2}\right) \cdot\left(z_{B 2}-z_{A 2}\right) \\
& d_{2}=\left(x_{B 2}-x_{A 2}\right) \cdot\left(z_{C 2}-z_{A 2}\right)-\left(x_{C 2}-x_{A 2}\right) \cdot\left(z_{B 2}-z_{A 2}\right) \\
& d_{1}=\left(x_{B 2}-x_{A 2}\right) \cdot\left(y_{C 2}-y_{A 2}\right)-\left(x_{C 2}-x_{A 2}\right) \cdot\left(y_{B 2}-y_{A 2}\right) .
\end{aligned}
$$

Transfer vector $P=\left(x_{O 2}, y_{O 2}, z_{O 2}\right)$ in the matrix of homogeneous transformation was found from equation (10). The direction cosines of the normal vector $n=\left(d_{1} ;-d_{2} ; d_{3}\right)$ of plane $O_{2} x_{2} y_{2} z_{2}$ are calculated as:

$$
a_{x}=\cos \alpha_{O 2 z}=\frac{d_{1}}{\sqrt{d_{1}^{2}+d_{2}^{2}+d_{3}^{2}}} ; a_{y}=\cos \beta_{O 2 z}=\frac{-d_{2}}{\sqrt{d_{1}^{2}+d_{2}^{2}+d_{3}^{2}}} ; a_{z}=\cos \gamma_{O 2 z}=\frac{d_{3}}{\sqrt{d_{1}^{2}+d_{2}^{2}+d_{3}^{2}}}
$$


The elements $s_{x}, s_{y}, s_{z}$ of the rotation matrix around the axis $O_{2} y$ defined as the angle between the unit vectors of the axes $\mathrm{O}_{2} z$ and $\mathrm{O}_{2} x$, the last of which is coincide with the vector $\overrightarrow{\mathrm{O}_{2} \mathrm{C}_{2}}$ :

$$
\begin{aligned}
& s_{x}=\cos \alpha_{O 2 y}=\frac{x_{C 2}-x_{O 2}}{\sqrt{\left(x_{C 2}-x_{O 2}\right)^{2}+\left(y_{C 2}-y_{O 2}\right)^{2}+\left(z_{C 2}-z_{O 2}\right)^{2}}} ; \\
& s_{y}=\cos \beta_{O 2 y}=\frac{y_{C 2}-y_{O 2}}{\sqrt{\left(x_{C 2}-x_{O 2}\right)^{2}+\left(y_{C 2}-y_{O 2}\right)^{2}+\left(z_{C 2}-z_{O 2}\right)^{2}}} ; \\
& s_{z}=\cos \gamma_{O 2 y}=\frac{z_{C 2}-z_{O 2}}{\sqrt{\left(x_{C 2}-x_{O 2}\right)^{2}+\left(y_{C 2}-y_{O 2}\right)^{2}+\left(z_{C 2}-z_{O 2}\right)^{2}}} .
\end{aligned}
$$

The elements $n_{x}, n_{y}, n_{z}$ of the homogeneous transformations matrix characterize the rotation around the axis $O_{2} x$. These elements are calculated as a product of the unit vectors of axes $\mathrm{O}_{2} x$ and $\mathrm{O}_{2} z$ :

$$
\begin{aligned}
& n_{x}=\cos \alpha_{O 2 x}=s_{y} \cdot a_{z}-s_{z} \cdot a_{y} \\
& n_{y}=\cos \beta_{O 2 x}=s_{z} \cdot a_{x}-s_{x} \cdot a_{z} \\
& n_{z}=\cos \gamma_{O 2 x}=s_{x} \cdot a_{y}-s_{y} \cdot a_{x}
\end{aligned}
$$

Thus, the grouping of the expression (10), (13) - (19) the homogeneous transformations matrix is obtained. The basic constraints of the workspace of Hauff platform connected with the limited length of kinematic chains, as well as the absence of singular configurations in which the mechanism either losing one or more independent degrees of freedom, or appears uncontrollable mobility.

\section{Singular pose of Hauff platform}

Problems of analysis of the workspace and the loss of controllability are related to both mechanisms with serial and parallel structure. Singular poses of a mechanism refer to as the self-intersecting of a configuration manifold at the certain critical value of links parameters [9]. Detection singularities and ways to overcome them in the robots kinematics are described in the works of many Russian and foreign authors [1-8]. It should be noted that the extension of workspace without singularity loci is very complex problem in the synthesis and analysis of parallel mechanisms.

The analytical approach to study of singularities and similar to them pose of these mechanisms based on the concepts and methods of theoretical mechanics, illustrated in [9]. The singularities of parallel mechanisms were considered in many publications, for examples: Hunt [1], Merlet [2], Glosselin and Angeles [3], Parenti-Gastelli and Innocenti [4]. The singular loci can be determined via constrains equations for planar and spatial parallel mechanisms as it was done by professors Huang [5], Gosseling and Wang [6]. Russian scientists Glazunov and Rashoyan [7], Kraynev and Glazunov in paper [8] shown by means screw groups that singular configurations form (( $W$ - 1$)$-dimension) singular zone where $W$ is the degree of freedom.

In general, the virtual displacement of the moving platform of Hauff manipulator equals to the total differential of the radius vector respect to the generalized coordinates:

$$
\delta r_{i}=\sum_{j=1}^{6} \frac{\partial r_{i}}{\partial l_{j}} \delta l_{j}
$$

Number $i$ of the appropriate mobility is changed from 1 to 6 i.e. $i=1, \ldots, 6$. Critical values of the lengths of rods in which configuration manifolds (or set of possible positions) of the parallel mechanism have self-intersections correspond to zero value of the partial derivative of the function (20): 
$\delta r_{i}=0 \Leftrightarrow\left\{\begin{array}{l}\frac{\partial r_{i}}{\partial l_{A 1 A 2}}\left(l_{A 1 A 2}, l_{C 1 A 2}, l_{B 1 B 2}, l_{A 1 B 2}, l_{B 1 C 2}, l_{C 1 C 2}\right)=0 ; \\ \cdots \\ \frac{\partial r_{i}}{\partial l_{C 1 C 2}}\left(l_{A 1 A 2}^{*}, l_{C 1 A 2}^{*}, l_{B 1 B 2}^{*}, l_{A 1 B 2}^{*}, l_{B 1 C 2}^{*}, l_{C 1 C 2}^{*}\right)=0 .\end{array}\right.$

In case of the ideal permanent links in the studied parallel mechanism, the system of equations (21) permits to evaluate the following facts:

- the system has one or several of isolated solutions;

- the limit values of the generalized coordinates and as a consequence the border of the workspace of Hauff Platform.

Using the properties of the Jacobian matrix for the analysis of the workspace of the studied parallel mechanism is not possible due to the absence the position function in explicit form. Therefore screw theory is more appropriative method to study singularities loci in the researched mechanism.

\section{An analytical approach to calculating the amount of workspace platform Hauff}

Analysis of the workspace of the mechanism will be to carry out, on the assumption that the points on a border of the separated singularities loci are viewed as unattainable. As a datum will be bring the origin of stationary coordinate system $O_{1} x_{1} y_{1} z_{1}$ connected with base platform $A_{l} B_{l} C_{l}$. Matrix of Plucker co-ordinates consists of the unit screws directed along the bars of studied parallel mechanism is written as follows:

$E=\left(\begin{array}{llllll}x_{A_{1} A_{2}} & y_{A_{1} A_{2}} & z_{A_{1} A_{2}} & x_{A_{1} A_{2}}^{0} & y_{A_{1} A_{2}}^{0} & z_{A_{1} A_{2}}^{0} \\ x_{C_{1} A_{2}} & y_{C_{1} A_{2}} & z_{C_{1} A_{2}} & x_{C_{1} A_{2}}^{0} & y_{C_{1} A_{2}}^{0} & z_{C_{1} A_{2}}^{0} \\ x_{A_{1} B_{2}} & y_{A_{1} B_{2}} & z_{A_{1} B_{2}} & x_{A_{1} B_{2}}^{0} & y_{A_{1} B_{2}}^{0} & z_{A_{1} B_{2}}^{0} \\ x_{B_{1} B_{2}} & y_{B_{1} B_{2}} & z_{B_{1} B_{2}} & x_{B_{1} B_{2}}^{0} & y_{B_{1} B_{2}}^{0} & z_{B_{1} B_{2}}^{0} \\ x_{B_{1} C_{2}} & y_{B_{1} C_{2}} & z_{B_{1} C_{2}} & x_{B_{1} C_{2}}^{0} & y_{B_{1} C_{2}}^{0} & z_{B_{1} C_{2}}^{0} \\ x_{C_{1} C_{2}} & y_{C_{1} C_{2}} & z_{C_{1} C_{2}} & x_{C_{1} C_{2}}^{0} & y_{C_{1} C_{2}}^{0} & z_{C_{1} C_{2}}^{0}\end{array}\right)$

Plucker coordinates of unit screw $E_{A 1 A 2}$ collinear to $\operatorname{rod} A_{1} A_{2}$ (the first row of matrix (22)) are calculated the following way:

the vector directed along bar $A_{1} A_{2}$ is described through the coordinates:

$\left(\begin{array}{lll}x_{A_{1} A_{2}} & y_{A_{1} A_{2}} & z_{A_{1} A_{2}}\end{array}\right)=i \cdot \frac{\left(x_{A 2}-x_{A 1}\right)}{l_{A_{1} A_{2}}}+j \cdot \frac{\left(y_{A 2}-y_{A 1}\right)}{l_{A_{1} A_{2}}}+k \cdot \frac{\left(z_{A 2}-z_{A 1}\right)}{l_{A_{1} A_{2}}}$

the coordinates of the moment are derived as a result of the cross product of the radius vector $\overrightarrow{O A_{2}}$ and the unit vector $\overrightarrow{A_{1} A_{2}}$ :

$$
\begin{aligned}
& \left(\begin{array}{lll}
x_{A_{1} A_{2}}^{0} & y_{A_{1} A_{2}}^{0} & z_{A_{1} A_{2}}^{0}
\end{array}\right)=\left(\begin{array}{c}
x_{A 1} \\
y_{A 1} \\
z_{A 1}
\end{array}\right) \times\left(\frac{\left(x_{A 2}-x_{A 1}\right)}{l_{A_{1} A_{2}}} \frac{\left(y_{A 2}-y_{A 1}\right)}{l_{A_{1} A_{2}}} \frac{\left(z_{A 2}-z_{A 1}\right)}{l_{A_{1} A_{2}}}\right)= \\
& =i \cdot\left(y_{A 1} \cdot\left(z_{A 2}-z_{A 1}\right)-z_{A 1} \cdot\left(y_{A 2}-y_{A 1}\right)\right)-j \cdot\left(x_{A 1} \cdot\left(z_{A 2}-z_{A 1}\right)-z_{A 1} \cdot\left(x_{A 2}-x_{A 1}\right)\right)+ \\
& +k \cdot\left(x_{A 1} \cdot\left(y_{A 2}-y_{A 1}\right)-y_{A 1} \cdot\left(x_{A 2}-x_{A 1}\right)\right) .
\end{aligned}
$$

Plucker co-ordinates of other unit screws are obtained the similar way. When matrix (22) is singular, two or more kinematic screws will be located in the same plane and studied mechanism will have a singular pose. Physically, this means that the vectors are linearly independent and there is uncontrolled mobility.

According to the screw theory [11] one more attribute of the singular poses is equal to zero of the relative moments of 
unit vectors which intersect at the joints of the moving platform of Hauff manipulator. Using matrix (22) the equations for analyze of the workspace of studied mechanism are written as follows:

$$
\begin{aligned}
& \operatorname{mom}\left(E_{A 1 A 2}, E_{C 1 A 2}\right)=x_{A 1 A 2} x_{C 1 A 2}^{0}+y_{A 1 A 2} y_{C 1 A 2}^{0}+z_{A 1 A 2} z_{C 1 A 2}^{0}+x_{A 1 A 2}^{0} x_{C 1 A 2}+y_{A 1 A 2}^{0} y_{C 1 A 2}+z_{A 1 A 2}^{0} z_{C 1 A 2}=0 \\
& \operatorname{mom}\left(E_{A 1 B 2}, E_{B 1 B 2}\right)=x_{A 1 B 2} x_{B 1 B 2}^{0}+y_{A 1 B 2} y_{B 1 B 2}^{0}+z_{A 1 B 2} z_{B 1 B 2}^{0}+x_{A 1 B 2}^{0} x_{B 1 B 2}+y_{A 1 B 2}^{0} y_{B 1 B 2}+z_{A 1 B 2}^{0} z_{B 1 B 2}=0 \\
& \operatorname{mom}\left(E_{B 1 C 2}, E_{C 1 C 2}\right)=x_{B 1 C 2} x_{C 1 C 2}^{0}+y_{B 1 C 2} y_{C 1 C 2}^{0}+z_{B 1 C 2} z_{C 1 C 2}^{0}+x_{B 1 C 2}^{0} x_{C 1 C 2}+y_{B 1 C 2}^{0} y_{C 1 C 2}+z_{B 1 C 2}^{0} z_{C 1 C 2}=0
\end{aligned}
$$

\section{Conclusion}

In the article the direct kinematics problem for Hauff manipulator have been solved in the form of a homogeneous transformation matrix. The advantages of the using approach consist in the same type of equations (1 - 9) and as a consequence the same type of non-linearity in all of equations. In particular, it was used the Euclidean distance between the joints of the moving and base platform.

Considered in the article, the problem of singular poses of the parallel mechanisms is especially important in the synthesis of manipulative mechanisms and its control. Screw theory has been used for analysis of singular pose due to more effectiveness as compared with the Jacobian matrix. In this case it does not require the constraint equations in explicit form and their derivative respect to the generalized coordinates.

As a result of the workspace analysis, it was determined that the theoretical displacement volume will be reduced by $4.48 \%$ if points in the singular loci considered to be achievable. The total loss will be achieved by $4.69 \%$ if the zero values of relative moments are applied.

Despite the fact that the total volume loss of the workspace of the studied parallel mechanism did not exceed $5 \%$, it should be noted that the analysis of the singular loci excluding degenerating links reduces the evaluation in more than 17 times.

\section{References}

[1] K. Hunt. Structural kinematics of in-parallel-actuated robot arms, ASME Journal of Mechanism, Transmissions, and Automation in Design 105 (4) (1983) 705 - 712.

[2] J.P Merlet. Singular configurations of parallel manipulators and Grassman geometry, The international Journal of Robotics Research 8(5) (1989) 45 - 56.

[3] C. M. Gosselin, J. Angeles. Singularity analyses of close loop kinematic chains, IEEE Transactions of Robotics and Automation 6(3) (1990) $281-290$.

[4] V. Pareti-Castelli, C. Innocanti. Direct displacement analisys for some classes of spartial parallel mechanism. Proceedings VIII CISM-IFToMM Symposium on Theory and Practice of Robots and Manipulators, Cracow, Poland, 1990, pp. 123-130.

[5] Z. Huang. The kinematics and type synthesis of lower-mobility parallel robot manipulators. Proceedings XI World Congress in Mechanism and Machine Sciense. Tainjin, China, 2004, pp. 65-76.

[6] C. Gosselin, J. Wang. Singularity loci of planar parallel manipulators with revolute actuators, Robotics and Automation System 21 (1997) 377 - 398.

[7] V. Glazunov, G. Rashoyan. The directions of motion of 1-co-ordinate manipulators from special configurations, Journal of Machinery (7) (1990) pp. 9-12 .

[8] A. Krainev, V. Glazunov. Design and analisys of spartial mechanisms with parallel structure. Proceedings VIII World Congress on TMM, Prague, Czechoslovakia, 1991, pp. $105-108$.

[9] V. Samsonov. Restructurong of configuration mainfolds and critical systems. Journal of PMM, (63) (1999) - pp. 770-774.

[10] A. Lapikov, V. Pashchenko. Solution of the direct kinematics problem for Hugh Stewart platform using analytical equations plane . Journal of Science and education. (2014).

[11] F. Dimentberg Theory of screws and its applications. (1978). - $328 \mathrm{pp}$

[12] Rat N.-R., Neagoe M., Godu C., Stan S.D. Dynamic Analysis of an Isoglide 3-T3 Parallel Robot, Annals of DAAAM for 2009 \& Proc. of the 20th International DAAAM Symposium, P. 15-16.

[13] Angeles, J.(2003),Fundamentals of Robotic Mechanical Systems, Springer.

[14] B. Andrievsky et al. Control of 6-DOF Stewart platform on the basis of pneumatic. Proceedings XII Conference on theory of control (2014) pp. 2150 - 2153. 